\title{
Clopidogrel-Induced Interstitial Lung Disease: A Case Report
}

\author{
Jin $A n^{\prime}$ \\ Seung Hwan Lee ${ }^{2}$ \\ Boksoon Chang (1D) \\ 'Department of Pulmonary, Allergy and \\ Critical Care Medicine, Kyung Hee \\ University Hospital at Gangdong, College \\ of Medicine, Kyung Hee University, Seoul, \\ Republic of Korea; ${ }^{2}$ Department of \\ Neurosurgery, Stroke and Neurological \\ Disorder Center, Kyung Hee University \\ Hospital at Gangdong, College of \\ Medicine, Kyung Hee University, Seoul, \\ Republic of Korea
}

\begin{abstract}
In patients, with cerebral infarction resulting from intracranial arterial stenosis, the combined administration of clopidogrel and aspirin may be needed for to prevent subsequent ischemic attacks. Clopidogrel has an inevitable adverse effect profile, and the most common complications are related to hemorrhagic propensity. A 79-year-old female patient had used aspirin (100 $\mathrm{mg}$ /day) for cerebral infarction and then a dual antiplatelet regimen of aspirin and clopidogrel $(75 \mathrm{mg} /$ day) because of severe stenosis in both anterior cerebral arteries. Two weeks later, the patient presented with dyspnea started 3 days ago, which had worsened in the last 24 hours. Chest computed tomography on admission showed symmetric peribronchial ground-glass opacity with reticulation in both lungs. Microorganism tests, including serology and bronchoalveolar lavage for infection, were all negative. Clopidogrel was withdrawn because of suspected clopidogrel-induced interstitial lung disease, and steroid treatment was initiated. Clinical signs and chest radiographs improved after steroid treatment, and she was discharged on day 21 of admission. This case report shows that clopidogrel can induce interstitial lung disease as a rare complication and underscores the importance of recognizing this adverse effect in clinical practice.
\end{abstract}

Keywords: clopidogrel, cerebral infarction, interstitial lung disease, drug-induced lung disease

\section{Introduction}

Clopidogrel is a drug widely use for the secondary prevention of atherothrombotic complications in patients with myocardial infarction and ischemic stroke. ${ }^{1}$ This drug inhibits platelet aggregation - increasing bleeding time and reducing blood viscosity - by inhibiting the action of adenosine diphosphate (ADP) on platelet receptors. $^{2}$ The adverse effects of clopidogrel are similar to those of other antiplatelet agents, such as ticlopidine or aspirin, and the most common complication is gastrointestinal bleeding. ${ }^{3,4}$ However, clopidogrel has not been known to induce interstitial lung disease (ILD). Here, we report a case of the rare adverse effect of clopidogrel-induced ILD in a patient with cerebral infarction resulting from intracranial arterial stenosis.

\section{Case Report}

A 79-year-old woman with prior cerebral infarction in the left basal ganglia presented with headache and dizziness. Magnetic resonance imaging revealed an unruptured aneurysm on the right superior cerebellar artery (SCA). She had been taking an aspirin $(100 \mathrm{mg} / \mathrm{d})$ and atorvastatin $(40 \mathrm{mg} / \mathrm{d})$ since the occurrence of cerebral infarction, 
amlodipine $(5 \mathrm{mg} / \mathrm{d})$ and telmisartan $(40 \mathrm{mg} / \mathrm{d})$ for hypertension, and metformin $(1700 \mathrm{mg} / \mathrm{d})$ for type 2 diabetes mellitus. Her hypertension was uncontrolled before cerebral infarction, but it became controlled after taking the medication for hypertension. The patient never smoked and had no history of autoimmune diseases or allergic reactions.

Cerebral angiography revealed a small SCA aneurysm with a broad neck, with a maximum diameter of $2.62 \mathrm{~mm}$ and a neck diameter of $2.73 \mathrm{~mm}$. In addition, there was narrowing of both anterior cerebral arteries (ACA), which could lead to extensive cerebral infarction. Because the unruptured SCA aneurysm was small and unlikely to rupture, it was determined to be followed with periodic brain imaging. A dual antiplatelet regimen of aspirin $(100 \mathrm{mg} / \mathrm{d})$ and clopidogrel $(75 \mathrm{mg} / \mathrm{d})$ was used because of severe stenosis of the ACA.

Two weeks later, the patient was admitted to the hospital due to dyspnea that started 3 days prior. She complained of anorexia, nausea, dyspnea on exertion, and chest pain. There was no complaint of cough, sputum, rhinorrhea, or chills. On admission, she was hypoxemic (room air pulse oximetry,
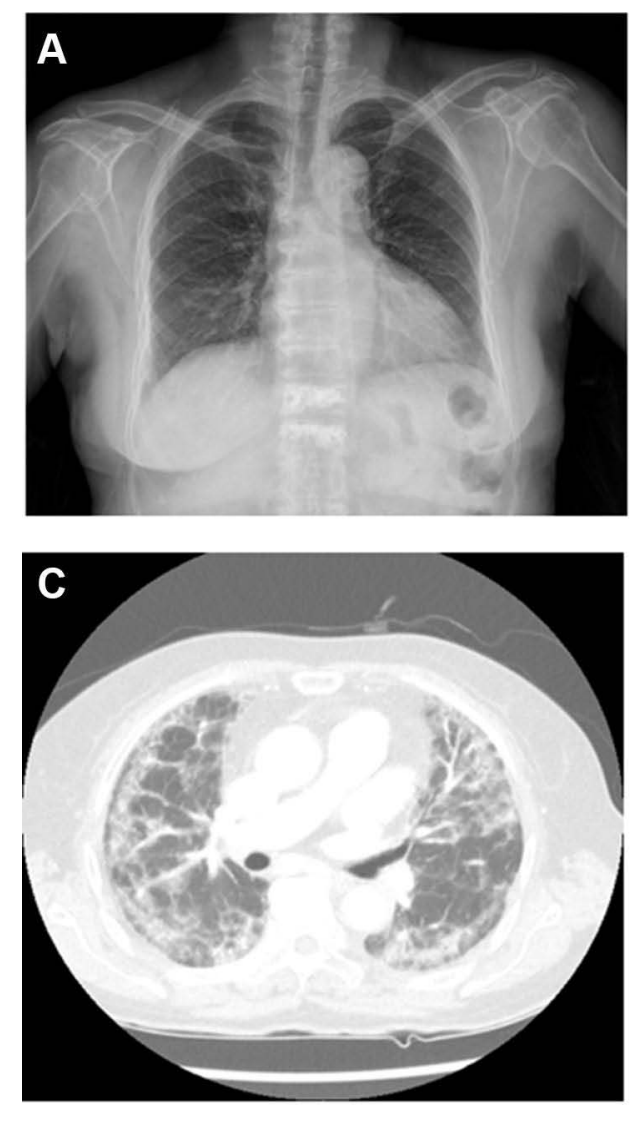

91\%), and chest radiography revealed features of multifocal consolidation and reticulonodular opacities in both lungs (Figure $1 \mathrm{~A}$ and $\mathrm{B}$ ). The patient presented the following vital signs: blood pressure, $136 / 66 \mathrm{mmHg}$; heart rate, 78 beats/min; respiratory rate, 20 breaths/min; and body temperature, $36.7^{\circ} \mathrm{C}$. The results of arterial blood gas analysis were the following: $\mathrm{pH}, 7.422$; $\mathrm{PaCO} 2,38.3 \mathrm{mmHg} ; \mathrm{PaO}_{2}$, $57 \mathrm{mmHg} ; \mathrm{SaO}_{2}, 88.1 \%$ (2 L/min of oxygen with a nasal prong). Laboratory test results were: hemoglobin, $11.5 \mathrm{~g} / \mathrm{dL}$; white blood cell count, 7410 cells/ $\mu \mathrm{L}$ (neutrophils, 65.1\%; lymphocytes, $24.3 \%$; monocytes, $9.0 \%$; eosinophils, $0.9 \%$; and basophils, $0.7 \%$ ); platelet count, 327,000 cells $/ \mu \mathrm{L}$; and C-reactive protein, $1.6 \mathrm{mg} / \mathrm{dL}$. The following serum biochemistry results were within normal limits: aspartate aminotransferase, $19 \mathrm{IU} / \mathrm{L}$; alanine aminotransferase, $5 \mathrm{IU} / \mathrm{L}$; total bilirubin, $0.7 \mathrm{mg} / \mathrm{dL}$; alkaline phosphatase, $59 \mathrm{IU} / \mathrm{L}$; total protein, $7.0 \mathrm{~g} / \mathrm{dL}$; albumin, $3.5 \mathrm{~g} / \mathrm{dL}$; blood urea nitrogen, $7 \mathrm{mg} / \mathrm{dL}$; and creatinine, $0.57 \mathrm{mg} / \mathrm{dL}$. Forced expiratory volume in one second and forced vital capacity were 1.02 L (predicted, 75\%) and $1.07 \mathrm{~L}$ (predicted, 52\%),
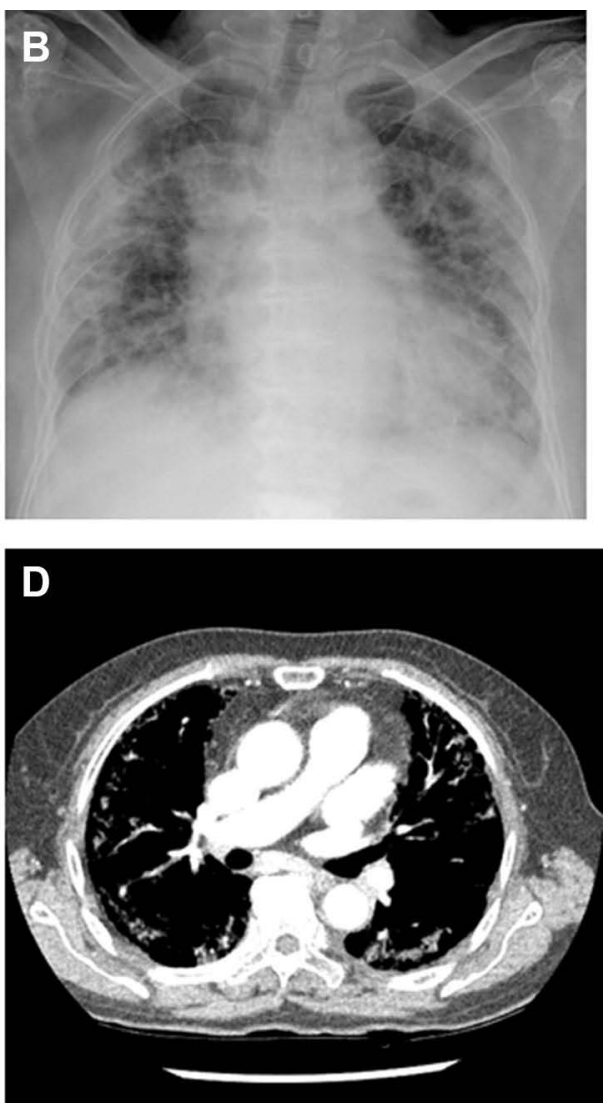

Figure I Chest radiography findings. Chest X-rays at 2 months before (A) and at admission (B). Lung window (C) and mediastinal window (D) on chest computed tomography on admission. The chest radiograph was normal at 2 months before admission (A). The chest radiograph on admission (B) demonstrates symmetric multifocal consolidation and reticulonodular opacities in both lungs. Axial lung window image (C) shows peribronchial ground-glass opacity (GGO), reticulation and consolidation in the periphery of both lungs. There was no significant endobronchial lesion and no axillary lymph node enlargement. 
respectively, and carbon monoxide diffusion capacity was $2.6 \mathrm{~mL} / \mathrm{mmHg} / \mathrm{min}$ (predicted, 19\%). Congestive heart failure was excluded because of normal ultrasound cardiography results and a normal level of serum brain natriuretic peptide.

Empirical antibiotics (piperacillin/tazobactam 4.5 g every 6 hours plus levofloxacin $75 \mathrm{mg}$ every 24 hours) were administered because bacterial pneumonia was not ruled out based on chest radiography findings. Enhanced chest computed tomography (CT) revealed symmetric peribronchial ground-glass opacity (GGO), reticulation, and consolidation in both lungs (Figure $1 \mathrm{C}$ and $\mathrm{D}$ ).

The patient had fever $\left(37.8^{\circ} \mathrm{C}\right)$ on the second day of hospitalization. In addition, oxygen saturation could not be maintained at $5 \mathrm{~L} / \mathrm{min}$ via nasal prong, and oxygen was administered via a high-flow nasal cannula $(30 \mathrm{~L} / \mathrm{min}$; $\mathrm{FiO}_{2}, 35 \%$ ). On day 4, bronchoalveolar lavage (BAL) was performed to rule out diffuse alveolar hemorrhage and atypical pneumonia. Bacterial polymerase chain reaction (PCR), viral PCR, Cytomegalovirus PCR, Pneumocystis jirovecii PCR, fungal culture, and acid-fast bacilli smear were performed in BAL samples. BAL samples revealed a clear color fluid, and no microorganisms were detected from any of the examinations. Nonetheless, patient's clinical symptoms continued to deteriorate.

One day 5, and clopidogrel-associated ILD was suspected after ruling out all other possible causes of her symptoms. We changed dual antiplatelet regimen of aspirin and clopidogrel to aspirin and initiated intravenous treatment with $1 \mathrm{mg} / \mathrm{kg} /$ day of methylprednisolone. Clinical signs and chest X-ray improved after clopidogrel withdrawal and steroid treatment (Figure 2A). The patient was discharged with oral prednisolone ( $25 \mathrm{mg}$ /day) on day 21 of hospitalization.
The steroid dose was tapered over 6 months in the outpatient setting. A follow-up chest radiograph at 6 months after discontinuation of steroid treatment showed no recurrence, and the patient's health status was good (Figure 2B).

\section{Discussion}

We report a case of ILD that occurred after clopidogrel administration and showed features of interstitial pneumonia on chest CT. These manifestations resolved after withdrawal of the culprit drug and treatment with prednisolone.

Clopidogrel is used to prevent secondary atherothrombotic events. ${ }^{2}$ The most common adverse effect is increased risk of bleeding. Cases of clopidogrel-induced acute lung injury are rare and associated with diffuse alveolar hemorrhage. ${ }^{5}$ In the study of patients at risk of ischemic events, fewer patients (4.5\%) reported dyspnea in the clopidogrel + aspirin group than in the placebo + aspirin group. ${ }^{4}$ Clopidogrel can potentially cause noncardiogenic pulmonary edema. ${ }^{6,7}$ However, chest CT showed a type of ILD in our patient.

The global incidence of drug-induced interstitial lung disease (DILD) is not clearly known, but epidemiological data from European countries have reported that $2.5-3 \%$ of ILD cases are drug induced. ${ }^{8,9}$ Anti-cancer drugs are the leading cause of DILD, accounting for $23-51 \%$ of cases, followed by disease-modifying anti-rheumatic drugs, antibiotics, nonsteroidal anti-inflammatory agents, psychiatric medications, and anti-arrhythmic agents. ${ }^{10}$ ILD induced by antiplatelet agents is rare, and most cases are secondary to aspirin and ticlopidine use.

The mechanism of DILD remains unclear but seems to be due to allergic reactions or neutrophil activation rather than direct toxicity from chemicals. ${ }^{11,12}$ Since clopidogrel
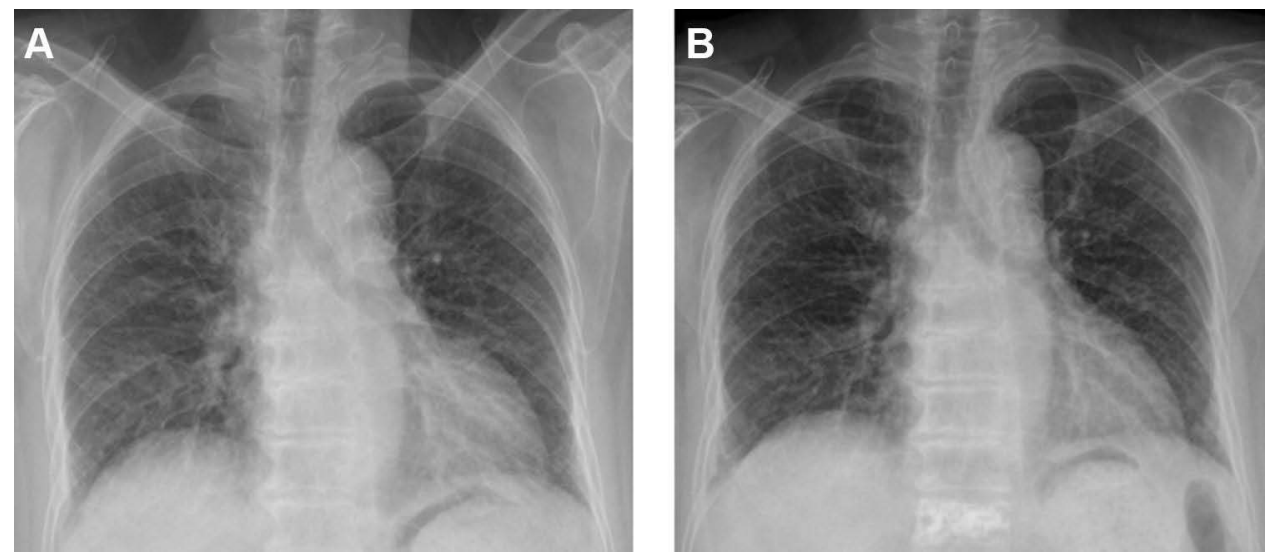

Figure 2 Chest X-ray on discharge (A) and at 6 months after discontinuation of steroid therapy (B). Note the improvement in multifocal consolidation and reticulonodular opacities in both lungs on the chest radiograph on hospital discharge and no clinical deterioration at 6 months after discontinuation of the steroid therapy. 
structurally and functionally resembles ticlopidine, a thienopyridine antiplatelet drug, ${ }^{13}$ chest CT findings for ILD induced by these two drugs were similar. Previous cases of ticlopidine-induced lung injury showed bronchiolitis obliterans organizing pneumonia, with aggressive symptoms and a longer recovery time. ${ }^{14,15}$ The pattern of cryptogenic organizing pneumonia in our patient was similar to that of ticlopidine-induced ILD. In these cases, ILD may be caused by drug allergic reactions because discontinuation of the culprit drugs and administration of corticosteroids lead to remission. ${ }^{16}$ In addition, ticlopidine and clopidogrel may activate neutrophils, suggesting that these agents contribute to the pathogenesis of lung injury. ${ }^{15}$ Since knowledge regarding immune responses to drugs is limited, further studies on the mechanisms underlying the initiation and progression of DILD are needed.

Patients at risk of adverse pulmonary events on drugs cannot be predicted based on drug history and risk factors. The risk factors for DILD vary depending on patient characteristics and drug therapy. Common risk factors include old age, smoking, and pre-existing lung disease, but drug dosage and drug interactions are risk factors for certain drugs. ${ }^{10,17}$ The diagnosis of DILD is challenging because of the heterogeneity of risk factors. In our patient, DILD was not detected earlier because she had no specific risk factors, except for old age. Therefore, physicians should be familiar with possible DILD of medications they prescribe and be suspicious of DILD in symptomatic patients.

Diagnosis and management of DILD are challenging. In our patient, the diagnosis was based on (1) a history of drug exposure with lack of changes other than addition of clopidogrel, (2) clinical and imaging findings, (3) exclusion of other lung disease, and (4) clinical improvement after discontinuation of clopidogrel and use of glucocorticoid treatment. Other lung diseases, such as hypersensitivity pneumonitis and eosinophilic pneumonia, were ruled out by differential cell count in BAL sampled (lymphocytes, $8 \%$; eosinophils, 3\%). The observation of previous cases is essential for diagnosis; however, the number of reported cases of clopidogrel-induced ILD is small, and we did not recognize DILD earlier. There are lack of guidelines for managing DILD. The first step is withdrawing the causal drug. Acute episodes of DILD usually disappear 24-48 $\mathrm{h}$ after the causal drug discontinuation. ${ }^{17}$ We discontinued clopidogrel and started glucocorticoid treatment because the patient experienced respiratory distress with rapid deterioration.
Glucocorticoids were commonly used to suppress the inflammatory response and prevent the deposition of fibrotic tissue. Nonetheless, no studies have evaluated the effectiveness of glucocorticoid therapy associated with withdrawal of the causal drug. ${ }^{17}$

This case report has limitations. First, the diagnosis of DILD was not based on histopathological findings. In this context, surgical or transbronchial lung biopsy was not performed because of possibility of several complications including bleeding and deterioration of hypoxia. Notwithstanding, the diagnosis was based on drug history, exclusion of other lung diseases, and radiologic findings. Second, clopidogrel readministration for accurate diagnosis was not performed because of the probability of reoccurrence of serious adverse reaction similar to this event.

\section{Conclusion}

Identification of DILD is challenging because clinical, radiological, and histological findings are nonspecific. Therefore, physicians should recognize any possible correlations between medications and the development of inflammatory lung damage. This case report shows that patients receiving clopidogrel may develop ILD, although its occurrence is rare. This is a substantial finding considering the widely use of clopidogrel as an antithrombotic agent.

\section{Data Sharing Statement}

To protect patient confidentiality, the data supporting this case report are available from the corresponding author on reasonable request.

\section{Ethics Approval and Consent to Participate}

Written informed consent for publication of the case details included accompanying images was obtained from the patient. The study was approved by the research ethics committee of Kyung Hee University Hospital at Gangdong (IRB No. KHNMC 2020-07-027).

\section{Consent for Publication}

The patient gave written consent to publish this material.

\section{Funding}

We received no funding for this study.

\section{Disclosure}

The authors report no conflicts of interest in this work. 


\section{References}

1. Geeganage CM, Diener HC, Algra A, et al. Dual or mono antiplatelet therapy for patients with acute ischemic stroke or transient ischemic attack: systematic review and meta-analysis of randomized controlled trials. Stroke. 2012;43(4):1058-1066. doi:10.1161/ STROKEAHA.111.637686

2. Kirkpatrick AC, Vincent AS, Dale GL, Prodan CI. Clopidogrel use and smoking cessation result in lower coated-platelet levels after stroke. Platelets. 2020;31(2):236-241. doi:10.1080/09537104.2019.1609661

3. Nathan AS, Sen S, Yeh RW. The risk of bleeding with the use of antiplatelet agents for the treatment of cardiovascular disease. Expert Opin Drug Saf. 2017;16(5):561-572. doi:10.1080/ 14740338.2017.1315101

4. Gent M, Beaumont D, Blanchard J, et al. A randomised, blinded, trial of clopidogrel versus aspirin in patients at risk of ischaemic events (CAPRIE). Lancet. 1996;348(9038):1329-1339.

5. Kim Y, Lim J, Lim J, Kim S, Jung T, Choi W. Pulmonary alveolar hemorrhage after clopidogrel use for ST elevation myocardial infarction. Korean Circ J. 2013;43(7):497-499. doi:10.4070/ kcj.2013.43.7.497

6. Erdinler IC, Ucer E, Eksik A, Akyol A, Yazici S. Noncardiogenic pulmonary edema associated with clopidogrel: a serious but unexpected side effect of clopidogrel. Can $J$ Cardiol. 2007;23 (6):478-480. doi:10.1016/S0828-282X(07)70788-2

7. Sarrot-Reynauld F, Bouillet L, Bourrain JL. Severe hypersensitivity associated with clopidogrel. Ann Intern Med. 2001;135(4):305-306. doi:10.7326/0003-4819-135-4-200108210-00028

8. Coultas DB, Zumwalt RE, Black WC, Sobonya RE. The epidemiology of interstitial lung-diseases. Am J Respir Crit Care Med. 1994;150 (4):967-972. doi:10.1164/ajrccm.150.4.7921471
9. Thomeer MJ, Costabel U, Rizzato G, Poletti V, Demedts M. Comparison of registries of interstitial lung diseases in three European countries. Eur Respir J. 2001;18:114s-118s.

10. Skeoch S, Weatherley N, Swift AJ, et al. Drug-induced interstitial lung disease: a systematic review. J Clin Med. 2018;7(10):356. doi: $10.3390 / \mathrm{jcm} 7100356$

11. Rodriguez AB, Barriga C, De la Fuente M. Effects of acetylsalicylic acid on the phagocytic function of human polymorphonuclear leukocytes in vitro. Gen Pharmacol. 1989;20(2):151-155. doi:10.1016/ 0306-3623(89)90007-4

12. Bednar MM, Dooley RH, Tapanes R, Lublin JC, Gross CE. Ticlopidine augments luminol-dependent chemiluminescence in human neutrophils. J Biolumin Chemilumin. 1995;10(2):85-89. doi:10.1002/bio.1170100204

13. Phillips EJ, Knowles SR, Shear NH. Serum sickness-like reaction associated with clopidogrel. Br J Clin Pharmacol. 2003;56(5):583. doi:10.1046/j.0306-5251.2003.01918.x

14. Alonso-Martinez JL, Elejalde-Guerra JI, Larrinaga-Linero D. Bronchiolitis obliterans-organizing pneumonia caused by ticlopidine. Ann Intern Med. 1998;129(1):71-72. doi:10.7326/00034819-129-1-199807010-00022

15. Nakamura $R$, Imamura $T$, Onitsuka $H$, et al. Interstitial pneumonia induced by ticlopidine. Circ J. 2002;66(8):773-776. doi:10.1253/ circj.66.773

16. Matsuno O. Drug-induced interstitial lung disease: mechanisms and best diagnostic approaches. Respir Res. 2012;13:39. doi:10.1186/ 1465-9921-13-39

17. Schwaiblmair M, Behr W, Haeckel T, Markl B, Foerg W, Berghaus T. Drug induced interstitial lung disease. Open Respir Med J. 2012;6 (1):63-74. doi:10.2174/1874306401206010063
Therapeutics and Clinical Risk Management

\section{Publish your work in this journal}

Therapeutics and Clinical Risk Management is an international, peerreviewed journal of clinical therapeutics and risk management, focusing on concise rapid reporting of clinical studies in all therapeutic areas, outcomes, safety, and programs for the effective, safe, and sustained use of medicines. This journal is indexed on PubMed Central, CAS,
EMBase, Scopus and the Elsevier Bibliographic databases. The manuscript management system is completely online and includes a very quick and fair peer-review system, which is all easy to use. Visit http://www.dovepress.com/testimonials.php to read real quotes from published authors. 